Voix et Images

\title{
Les Vingt-six figures d'une rhétorique de soi
}

\section{Gilles Thérien}

Volume 2, numéro 3, avril 1977

Jean Éthier-Blais

URI : https://id.erudit.org/iderudit/200067ar

DOI : https://doi.org/10.7202/200067ar

Aller au sommaire du numéro

Éditeur(s)

Les Presses de l'Université du Québec

ISSN

0318-9201 (imprimé)

1705-933X (numérique)

Découvrir la revue

Citer cet article

Thérien, G. (1977). Les Vingt-six figures d'une rhétorique de soi. Voix et Images, 2(3), 321-333. https://doi.org/10.7202/200067ar d'utilisation que vous pouvez consulter en ligne.

https://apropos.erudit.org/fr/usagers/politique-dutilisation/ 


\title{
Les Vingt-six figures d'une rhétorique de soi
}

\author{
Courir les bras ouverts en direction d'un auteur \\ (soit pour l'étreindre, soit pour l'étouffer), n'est-ce \\ pas la véritable objectivité? \\ Jean Éthier-Blais
}

Nul ne songerait à complimenter Fischer-Dieskau sur l'excellence de son chant. Ainsi en va-t-il de l'écriture d'Éthier-Blais. La lecture du Dictionnaire de moi-même ${ }^{1}$ suggère de multiples réflexions (reflets) qu'on tentera ici de dévoiler. Selon la méthode d'Éthier-Blais? Que non! L'énergie manquerait à trop aimer ou trop haïr les rencontres du hasard d'une lecture. II est vrai que des morts seraient moins gênants. Mais on court tout de même le risque de les voir se tenir en ribambelle dans une mémoire divisée en amour et en haine. II reste la dissection, celle du corps du livre, avec le curieux espoir de trouver à la pointe du scalpel l'âme de l'auteur: formel-formol, cela ne convient pas plus. Se laisser aller au plaisir du texte, oui, surtout qu'un dictionnaire autorise tant de substitutions, de subtils amusements. Reconstruire, non le texte, mais l'intertexte, celui qui file entre deux mots, deux phrases quand le livre se pose ou que les yeux se ferment. Engendré par le texte, il n'est à l'abri ni de l'harmonie ni de la monstruosité. Mimésis. Le critique et l'analyste reproduisent en quelque sorte ce qu'ils ont élu un temps pour modèle. Pauvreté de la création qui renvoie les auteurs à l'Auteur et au Livre... et les scribes aux auteurs.

On donne à Dieu toutes sortes de noms ou qualités dont beaucoup ne sont que des métaphores, par exemple, lorsque la Bible l'appelle: «Lion de Juda "; ou "Rocher de mon espérance " [...] Seuls ont valeur scientifique les noms et concepts qui expriment une perfection pure, comme la vie, la vérité, le bien, etc. [...] Nos concepts de perfection pure ne nous font connaître Dieu que d'une façon très inadéquate: la Divinité dans ce qui la constitue en propre, reste un mystère radicalement inaccessible à notre intelligence: c'est "l'Ineffable" et en ce sens, l' «Inconnaissable". 
Théodore, le professeur Théodore Salandon de Mater Europa ${ }^{2}$ a, dans son passé, cultivé Jean de saint Thomas. Il s'est penché sur le mystère des Noms divins. Il connaît certainement l'analogie de proportionnalité propre. Dieu, de par sa perfection, peut se voir attribuer tous les noms (ou presque!). II n'en demeure pas moins inconnaissable même s'il se dit le Verbe, le Logos. En somme, Dieu est une sorte de sous-entendu du dictionnaire, un intertexte transcendantal. Tous les noms sont impropres à le définir mais n'en constituent pas moins une "via negativa ". Le Professeur Salandon le sait et Éthier-Blais aussi. Le «dieu» du Dictionnaire de moi-même illustre son mystère et par des métaphores et par des concepts de perfection pure.

S'enfermer dans un dictionnaire est donc un projet plus absurde qu'ambitieux. Le mot introduit entre lui et celui qui le profère un tiers personnage, l'auteur, sorte de verbe désincarné qui transcende tous les mots qu'il utilise. Mon Stendhal et celui de $M$. Éthier-Blais sont faits de cette même non-chair et ne coïncident probablement pas. L'autobiographie (de fait - puisque tout est autobiographique à un degré plus ou moins grand) est ce projet de faire coïncider le personnage-auteur et l'hommeauteur toujours par le truchement des mots qui n'auront d'autre fonction que de créer un troisième personnage, celui qui se confesse. Le projet d'Éthier-Blais tente, sinon d'échapper à cet infini jeu de miroirs, du moins d'accuser cette absurdité en retirant aux mots ce pouvoir magique de dire la réalité. Et c'est sous le dogme de cette trinité païenne (MOTS/ JE/MOl-qui-écris) que se déroule le Dictionnaire de moi-même, le «même» ne pouvant être compris que par référence à un «autre» comme il est dit dans le Parménide et le Sophiste. "Éthier-Blais" ne fait d'ailleurs pas partie de la sélection.

L'analogie établit une comparaison. On y retrouve la métaphore. L'intertexte « Jean Éthier-Blais " sous-tend vingt-six figures de lui-même, au nombre même des lettres de l'alphabet, où chacune de ces lettres est représentée par un seul mot, cristallisation évidente, choix absolu. On y retrouve, non pas des définitions de soi mais de fins miroirs qui cadrent l'élément sélectionné et l'exploite selon toutes les ressources de la rhétorique. Vingt-six tableaux d'une exposition entre lesquels il est permis de circuler, de prendre ses distances, de revenir examiner tel ou tel détail. Vingtsix masques, jamais levés, qui cachent tous une même figure.

1925 (1926)

[Le Scorpion] est le signe de l'anus qui correspond psychanalytiquement aux valeurs d'excrement: défection, expulsion, liquidation, restitution; il transforme, détruit, corrompt, recompose... Mais s'il correspond à l'anus avec sa charge de pulsions agressives, il correspond aussi au sexe avec sa puissance créatrice, fécondante. Ce signe est sous la tutelle de Mars et de Pluton, le "prince des ténèbres", symbole des profondeurs et des 
ténèbres de notre nuit originelle, surtout de nos régions infernales.

André Barbault Traité pratique d'astrologie

Quand Éthier-Blais écrit: «Je suis né sous le signe du Scorpion, de la famille des arthropodes ${ }^{3}$ " c'est tout un programme qu'il annonce! Ce texte qui se trouve sous la rubrique "Zodiaque" est en fait annoncé dès la première, "Amour»: “En moi, c'est le scorpion qui s'arrache à la boue et qui croit que le bonheur ce sera le feu. $O$ erreur ${ }^{4}$ !"Sa naissance fixée au 15 novembre 1925 rend compte de l'utilisation du zodiaque. En comparant, sur ce sujet, le texte de l'auteur à celui de n'importe quel traité d'astrologie, dont celui cité plus haut, on ne manquera pas de remarquer que le Scorpion sert de prisme à Jean Éthier-Blais. II permet d'étendre le registre de la description de soi et ne constitue en aucune façon une recherche ésotérique d'une définition de soi. Le zodiaque ne sert pas à comprendre mais à se faire comprendre.

La magie des nombres, à laquelle se réfère l'auteur, quoique de façon erratique ${ }^{5}$, nous a suggéré une approche particulière de cet essai. L'alphabet comprend 26 lettres. La vie racontée de Jean Éthier-Ḅlais est comprise entre la première et la dernière lettre, $A(1), Z(26)$. La première année d'existence de l'auteur porte sur deux années de calendrier: 1925 et 1926. C'est une infime erreur des dieux d'avoir introduit cette année 25 dans une vie qui aurait pu se dérouler si harmonieusement en 26 . En effet, le lien entre 1 et 26 est 9 , de même que la somme des quatre chiffres $1,9,2,6$. Or le 9 correspond à «Ironie "... ce qui ne manque pas d'àpropos. II n'est pas question de prétendre que cela a été voulu ou aurait pu l'être. Les Muses sont plus subtiles et les coïncidences ne sont fortuites que pour les esprits les plus obtus.

Il serait donc possible de lire un lien entre la date de la première année de vie et le projet de dictionnaire. La description que fait ÉthierBlais du scorpion appuie cette prétention. Animal qui «marche tête baissée et la queue en l'air ", il a du venin dans la queue; chez lui les poumons et la tête ne faisant qu'un, la respiration risque fort de suivre un rythme cérébral. II semble aussi que la morsure du scorpion soit son unique objectif: "Tout converge vers la pointe extrême et le venin, afin de le projeter au-dehors ${ }^{7}$." L'ironie qui est une arme dont Éthier-Blais admiet se servir, blesse aussi la victime: "elle doit rentrer chez elle, contente et sûre d'elle-même, sans avoir senti les flèches. Dans l'escalier, soudain, elle se pose une question. S'est-on moqué d'elle? A-t-elle eu raison? $\mathrm{Ne}$ l'aurait-elle donc pas emporté? La question jaillit, avec l'incertitude. Rien qu'une fissure, ce qui n'est pas peu. Cette maison ne sera plus jamais neuve ${ }^{8}$. Ainsi l'ironie comme le venin poursuit son chemin. La victime. est prise de vitesse. Le bourreau n'est ni heureux ni malheureux. C'est sa nature de blesser.

“Amour», «Ironie», «Zodiaque» forment la constellation de base qui éclaire tous les arrangements que suggère le livre. II y sera question 
d'amour et de haine, de mort, de plaisir et de celui qui, entre ces deux pôles, cherche à prendre la distance que confèrent l'ironie, le mépris. L'instinct exhibitionniste l'emporte sur la confession. Le savoir-vivre, les bonnes manières tamisent la sincérité crue. II reste ce qu'il faut de pudeur pour forcer une lecture plus attentive et une complicité entre les lignes. C'est le cas du vocable "Amour». II est inutile d'y chercher quelque révélation fracassante, quelque confidence inattendue... et pourtant! L'accumulation des "nous", des "on"; le mélange immédiat de l'amour et de la souffrance dès la seconde phrase ${ }^{9}$ ( (L'amour, en naissant, suscite ses détresses »), la réduction de la femme à un rôle de stimulus, la vengeance comme partie intégrante de l'amour, la réversibilité subite de l'amour en haine, voilà des confidences indirectes qui rendent encore plus grave l'aveu "plus je vieillis, moins j'aime ${ }^{10}$ ". Et pour ceux qui savent lire, quelle subtile vérité que le faux féminin de la grammaire: "Je faisais un soir des aveux à une belle personne. Je lui promettais mers et mondes. Elle me refusa..." "L'amour se présente comme un manque, comme l'aboutissement d'un processus de sublimation et finalement comme une figure de rhétorique. Pourtant la haine, le désespoir, le désir inassouvi sont bien présents. Ils annoncent la mort, celle qu'on doit attendre stoïquement, sans plaintes. Pulsions de vie, pulsions de mort. Si Éthier-Blais ne dit rien de bien neuf là-dessus, il sait évoquer habilement «l'écharde dans la chair» qu'on ne saurait nommer.

De même, l'essai se termine encore par un manque, figuré par le trou. “Où ira ce corps, avec ses humeurs et ses larmes? Dans un trou ${ }^{12}$." La dernière lettre de l'alphabet, le $Z$ qui marque le terme (alors qu'en grec, il signifie la vie) évoque une mort consentie, voulue, au niveau du langage, moins comme un dernier désir qu'en tant que suprême incantation. Si le rituel pouvait donc rendre moins pénible cette mystérieuse (et finale) initiation! La langue superbe, comme ce roi triste, évoque ses propres funérailles. "J'aimerais mourir en Tunisie, l'automne, lorsque le vent et la pluie se mêlent et frappent à toutes les portes. On m'envelopperait dans un drap, on me déposerait devant ma maison, dans la terre froide et mouillée... ${ }^{13}$, Et plus tard, à la manière d'un film de Bergman, on verra un jardinier ramasser pêle-mêle dans une brouette les restes du grand homme et des immondices: «Regardez-le qui s'éloigne, la démarche lourde, poussant devant lui, tout entremêlées, la vie et la mort. Porteur ignorant de mon destin, il disparaît avec un autre jour ${ }^{14}$." Même la mort ne saurait mettre un terme à la grandeur métamorphosée en humilité de celui, ignorant, qui transporte. Le rêve de pouvoir ne connaît pas de limites et Freud s'y reconnaîtrait, qui a écrit: “[L'artiste] a finalement conquis par sa fantaisie, ce qui n'avait existé que dans sa fantaisie: honneurs, puissance et amour des femmes ${ }^{15}$."

La rencontre de cet Alpha et de cet Oméga, de ces deux personnes en Dieu engendre la troisième personne. Amour, père-mère, et zodiaque, mort-pouvoir donne naissance à Ironie, l'esprit... de finesse: "Le détachement de l'ironie rend l'esprit heureux et subtil16. "C'est aussi la méthode 
de Socrate, dit-il, encore que ce dernier l'ait utilisée en un sens moins pervers, moins dévastateur. Ironie mordante, ironie qui blesse, ironie que le vieillissement transforme en mépris. Il est difficile de ne pas voir là le baromètre des pulsions de vie et de mort. Il ne sera pas étonnant de voir, entés sur ces pulsions, deux autres reflets, «Bonheur» et “Désir». "Ai-je été heureux ${ }^{17}$ ?", il ne semble pas. L'utilisation du passé condamne le présent. Si l'amour est déçu, si la vie n'ouvre que sur une mort en brouette, le bonheur ne pourrait être que le sourire niais de celui qui ne comprend pas. Et pourtant, quelques pages plus loin, le désir se prend à rêver: "Que désire-t-on, vieux? La jeunesse. Revivre. [...] Renaître [...] Se retrouver Marquise de Pompadour, phtisique, après avoir été, deux siècles plus tard, écrivain montréalais ${ }^{18}$." Étrange désir qui choisit d'être la Pompadour malade plutôt qu'un Goethe en bonne santé! Derrière la surface des mots, la détresse pointe le museau. Désirer devenir centenaire et se trouver déjà vieux à cinquante ans quand il reste encore une autre moitié de vie devant soi, telle est la corde raide sur laquelle s'avance celui qui sait qu'il va tomber.

La trinité païenne est élaborée, symboliquement contenue dans le chiffre 1926. L'amour et la mort se partagent un “je" qu'agitent le désir, la détresse, la souffrance. L'homme nè marche pas vraiment. II est immobile sur le long ruban de la vie qui glisse sous lui.

Du passé, c'est mon enfance qui me fascine le plus; elle seule, à la regarder, ne me donne pas le regret du temps aboli. Car ce n'est pas l'irréversible que je découvre en elle, c'est l'irréductible...

Roland Barthes

«Ah! Mon père! pourquoi êtes-vous mort si tôt? [...] De vous, je n'ai que deux souvenirs ${ }^{19}$. "Que celui qui cherche à se définir passe par son père, voilà qui ést tout à fait normal. Le fils n'est-il pas toujours, en quelque sorte l'image du père? Si le lieu génétique est évident, le lien symbolique ne l'est pas moins. Le texte d'Éthier-Blais interpelle «Vous», celui qui n'est plus là, discours à l'au-delà, celui qui se résume en deux maigres souvenirs. Les références culturelles sont présentes: "Père, père, pourquoi m'avez-vous abandonné ? " Et cet abandon, aux mains de la mère, s'est, semble-t-il, transformé en véritable croix. "La vie sans vous? Je ne peux imaginer la mienne avec un homme à l'horizon, vers lequel me tourner, à qui parler, du même sexe que moi et géniteur. II me semble qu'il y a là contradiction ${ }^{20}$." La contradiction est une croix. Nietzsche ne parlait-il pas d'une contradiction de l'esprit à propos du dieu crucifié. Les contradictions logiques logent dans la chair. Le père mort, des femmes prendront la relève, des mères, l'une véritable, les autres plus ou moins fictives soit qu'elles habitent des textes, soit qu'oubliées par les révolu- 
tions, elles subsistent, anachroniques, dans le monde du capital. L'appel au père est exprimé comme un manque, un abandon et cela se répercute dans "Homme», "Jeunesse » et "Kyrie».

Si le père a manqué, si la mère est devenue la dépositaire de l'humanité, les pages qu'Éthier-Blais consacrent à l'Homme sont teintées d'un double héritage. L'homme en tant qu'espèce, abandonné par le géniteur (le père de l'espèce?) se porte bien mal. Il a connu, paraît-il, de meilleurs moments. De multiples faux pères ont fait leur apparition: "En cette fin de vingtième siècle, après Marx, Freud, Staline, Hitler, Mao, il faut craindre pour l'avenir. Quelle concentration soudain, de monstres ${ }^{21}$ !" II est intéressant de voir Freud côtoyer des penseurs politiques et des hommes de pouvoir. N'est-ce pas parce qu'il a mis le doigt sur les rapports de père à fils et de mère à fils, ébranlant ainsi l'antique sécurité familiale? L'homme de Jean Éthier-Blais est l'unité de masse du “déclin de l'Occident». Malgré les prédications de la mère, malgré l'enseignement (maternel) de la Bible, l'homme semble voué à une destruction que déplore l'auteur assis à sa table de travail. Entrouvrir la porte du Goulag, la refermer bien vite. Ensuite prendre un auteur classique, Homère par exemple, et chercher sur la page un destin meilleur. La mamelle sociale ne fournissant que du mauvais lait, il faut savoir opter pour la culturelle. Les mères, elles, ne manquent pas.

Aussi étonnant que cela puisse paraître, l'Homme n'est pas un reflet d'Éthier-Blais qui garde pour lui, un regard parfois affectueux, le plus souvent distant. L'humanisme classique fournit bien une valeur de remplacement mais à la condition de demeurer chez soi avec Chateaubriand, Madame de Staël et Napoléon. Quant à la rue grouillante, il faut y prendre garde: “Tenter de définir l'homme en 1975, c'est parler non plus d'un être de chair et d'os, mais d'une métastase sociale ${ }^{22}$."

Non saturé, le même discours réapparaît à propos de la « Jeunesse». Évidemment la jeunesse ne peut être que la période de pré-déclin. Les vieux singent la jeunesse parce qu'ils craignent la mort. Et les jeunes, eux, s'ils savaient ce qui les attend... Mais ils ne savent pas. Ils sont trop courageux, comme le jeune Éthier-Blais pratiquant les Exercices de saint Ignace et en même temps, trop ignorants. «J'ai honte d'être né, j'ai honte de mes semblables. Je ne veux pas être ange. Je ne veux rien. Je regrette, tout simplement, d'être sorti vivant du ventre de ma mère, d'avoir respiré une première fois, d'avoir vécu, de vivre, d'avoir à affronter une nouvelle, éternelle, vie ${ }^{23}$. " S'il méprise la jeunesse, c'est qu'il dit se mépriser. Et pourtant! La jeunesse se laisse prendre au vent de toutes les idéologies, lui pas. Il écrit: "Le monde inhabitable. La montée, contre nous, des jaunes, et des noirs... J'appartiens à la dernière génération à qui il sera donné de respirer à l'aise dans l'Occident. Déjà que de noirs effluves ${ }^{24}$ !" II met en garde contre le marxisme, il oublie le racisme. Il faut peut-être une sorte d'eugénisme qui assure aux grands esprits une postérité digne d'eux? Là encore, la mère domine, matrice universelle de toutes les races ou «sélectionneuse » d'extrême-droite. 
Père, jeunesse, Homme, ces décors d'une enfance québécoise de Sudbury ne pouvaient que mettre en scène le masochisme de la faute: «Kyrie», Ayez pitié. Quelle faute? Celle d'être là, comme personne, comme nation. Désespoir? Éthier-Blais serait un nationaliste désespéré pour qui, seule, la violence (celle du sang) est annonciatrice d'évolution ${ }^{25}$. II renie sans être Judas ni l'autre Jésus, Pierre Trudeau. II vilipende les politiciens véreux qui se disaient purs. Subitement on croit qu'Éthier-Blais a ouvert la porte, qu'il a sillonné les rues, que 1970 a été chez lui l'occasion d'un éveil encore plus grand de la désespérance, celle non seulement d'être une nation en voie de... disparition mais encore sauvée par des hommes qui n'ont pas la foi. Kyrie eleison, épisode de la messe du dimanche, de la tante soprano et de la mère alto. Kyrie eleison, les joies pures de la musique classique pendant que «le dimanche, les enfants s'ennuient», souvenirs d'une enfance bien ordonnée, peu traversée par l'aventure. Mère réelle, mère-patrie, la mère vient encore ici remplacer le manque créé par l'absence des pères. L'énergie, l'esprit de décision, le courage sont des vertus littéraires; la plainte, la récrimination, le découragement sont des modes de réaction à la réalité québécoise.

La lecture orientée vers la description subtile des pulsions de vie et de mort ne peut ici parler d'autre langage que celui de la pulsion de mort. La mort du père et ses échos dans la vie du fils marquent l'enfance décrite, le fait d'être né canadien-français non québécois avive peut-être le sens de la destruction de la race, destruction accompagnée d'un racisme envers les destructeurs. A tout cela, une seule solution: si nous avions du courage, c'est dans le sang qu'il faudrait renaître. La pulsion de vie est discrète, la pulsion de mort bat comme un tambour. A la réalité quotidienne, Éthier-Blais préfère celle de la littérature qui demande si peu d'oxygénation qu'on peut la pratiquer à l'agonie.

1967

Ce que vous voyez du monde, à travers cette fenêtre, est-il donc si beau que vous ne voulez à aucun prix regarder à travers une autre fenêtre, - et que vous essayez même d'empêcher les autres d'en faire la tentative?

Nietzsche

C'est sous le signe du regard que l'auteur se présente comme auteur. Les objets sont soigneusement choisis, de même que l'angle du regard: "Mon éditeur me téléphone. «J'ai reçu vos livres». Ce sont mes premiers ouvrages Signets I et II. Je cours chez lui, je tiens les deux livres dans mes mains, je sens vivre la page, sur elle palpiter mon nom. Je les feuillette. Je suis fier. Enfin, auteur! Je tremble, sors, apporte chez-moi ces précieux objets. Toute la journée, je les regarde, ému26." L'homme fait, c'est l'écrivain. Son regard se pose sur lui-même. Doit-il parfois trouver autre chose dans son champ de vision que la fenêtre, apparemment ouverte sur le monde, 
devient un miroir. Le regard s'intériorise. L'écrivain peut se permettre un voyeurisme silencieux.

La maturité de l'homme coïncide avec son existence d'écrivain. Le rappel ici de Signets / est important. Ce livre est surtout consacré à des écrivains français, hommage qu'Éthier-Blais souhaite rendre à la mèrepatrie de l'esprit, celle qui sera responsable en définitive de son style et de son bon goût. Les objets qu'il offre alors à son regard et au nôtre, seront teintés eux aussi de ce sentiment filial. L'histoire de la littérature française avec son portrait de Baudelaire, le cimetière de Passy, la Cour carrée du Louvre, les ruines d'Angkor Vat et l'hôtel où on s'habille en blanc, le portrait du Très Révérend Père Lacordaire, un être cher (encore l'ambiguïté grammaticale!) et sans oublier le cardinal Richelieu, avec Napoléon, deux héros sanguinaires les plus communs au Canada français.

Bien entendu, les objets sur lesquels se pose le regard d'ÉthierBlais sont des prétextes. Pris séparément, ils n'ont qu'une valeur bien relative mais dans le réseau tissé par l'auteur, ils deviennent des miroirs, des reflets. Les figures du "Regard", de la "Fenêtre», de la "Solitude» et de l' "Exil" sont les quatre coins de la toile que tisse l'écrivain. Le regard n'est pas que le reflet de l'âme, il est aussi l'image du désir et alors l'écrivain devient un voyeur. L'œil regarde et est regardé. II sert, tout autant que les mots, à mentir. Voir et regarder, c'est apprendre à choisir. Même la fenêtre ment. Elle découpe un espace en dehors et en dedans mais sa vitre peut être un miroir. Le regard engendre la solitude soit par une extrême sélection de l'objet: "L'oiseau, que de ma fenêtre je regarde sautiller d'une branche à l'autre, l'œil sec, il est seul. Plus on s'abstrait, plus la solitude vous gagne"; soit par un retour sur soi: "Lorsque j'écoute vraiment, je ne souris pas. J'ai l'air ennuyé, je plisse le front, j'ai le teint jaune. C'est que d'écouter m'oblige à m'arracher à moi-même ${ }^{27}$."

II est possible à l'cil de contempler quelque paysage intérieur: la solitude de Madame Récamier à l'Abbaye-aux-Bois, ou bien celle de Chateaubriand ou de Napoléon. L'exil est aussi une forme de solitude, qui peut être évoqué en passant sans transition de Coppet à Hammamet. Vaincre la solitude, l'exil, c'est savoir utiliser son regard intérieur. C'est recréer le passé dans le présent grâce au mur de la caverne où s'agitent les formes éternelles (et belles) d'Héliodore et de Sosthène.

Pourquoi écrire? L'écriture est absente comme figure de l'alphabet d'Éthier-Blais. Mais elle anime ce dictionnaire, autrement sans vie. «Écrire! Quoi donc? Et puis après? Qu'ai-je à dire? Je ne sais. Je suis poussé par une force qui fait que je ne suis heureux et malheureux que le stylo à la main, assis à une table, en train de raconter quelque chose ${ }^{28}$." Le regard n'est en somme que la direction de l'écriture, la fenêtre, sa limite. L'écriture est, elle, solitude et exil. Quoi écrire? La lecture du Dictionnaire de moi-même invite à croire que cela n'est pas très important. On y retrouve l'affirmation tacite que non seulement le style est l'homme mais encore qu'il le fait. Étrange paradoxe où la vanité côtoie la modestie, celle d'admettre superbement qu'on n'a peut-être bien rien à dire. 
Il faut que tu saches que, nous autres poètes, nous ne pouvons suivre le chemin de la beauté sans qu'Éros se joigne à nous et prenne la direction; encore que nous puissions être des héros à notre façon et des gens de guerre disciplinés, nous sommes comme les femmes, car la passion est pour nous édification, et notre aspiration doit demeurer amour... tel est notre plaisir et telle est notre honte.

Thomas Mann

Le poids de l'œuvre se fait sentir. On ne sait plus très bien si c'est l'œuvre qui garantit l'auteur ou l'auteur... Elle avance à petits pas. Le Dictionnaire de moi-même se veut un essai autobiographique. De quelle biographie? Celle de l'auteur? Celle de Jean Éthier-Blais? C'est le «il décrit son univers [...] dans le mystère de l'écriture" de la couverture qui retient l'attention. Quel mystère? Le mot Vérité va-t-il donner la clé? Non. La vérité, dont il est ici question est celle de la religion. C'est la question posée à Pilate. Rousseau promettait de dire toute la vérité sur lui-même. La Rochefoucauld se décrit tel qu'il croit être. Éthier-Blais parle de la vérité de Dieu, de la vérité qui s'enseigne, transmise par l'éducation catholique. Est-ce à dire qu'il ne reste plus de place pour sa petite vérité à soi, celle qui peut être même un mensonge - à condition qu'il y ait sincérité.

Dogme, vérité, enseignement, tels sont les boulets dont s'affuble en 1976 Jean Éthier-Blais. Si l'écriture opère une distance, elle devient en même temps un sujet de honte. La libération est loin. La pulsion de mort est plus présente encore une fois que la pulsion de vie. La liberté, absente de son alphabet au profit de «Lecture», est envisagée sous l'angle de l'ordre. Puisque la vérité est carcan, la liberté se travestit en ordre. L'ordre social bien sûr. Le cadre, le camp, la loi, l'exclusion, l'esclave, la hiérarchie. Timidement, l'auteur s'inquiète: «Plume à la main, j'avance dans de vastes forêts. Est-ce là que se terre l'ordre de mon bonheur? Je ne sais." Plus haut, sur le ton de la confidence: "Pourquoi ne pas le dire? Je crois que tout est désordre et nuit ${ }^{29}$. " Le désordre est érotique, il retient les corps alors qu'on voudrait s'échapper vers l'esprit. L'ordre est aussi moral, imposé par les institutions familiale et sociale. A la contrainte collective s'ajoute la contrainte individuelle. L'ordre passe par les jésuites, la Révolution, c'est-à-dire la guillotine. L'ordre accompagne les différents pouvoirs. Refuser l'ordre sans prendre les armes, c'est faire le mort.

Le refuge pour celui qui fait le mort, qui anticipe le désordre, c'est la nuit. "Je n'aime la nuit que pour le sommeil et ses plaisirs, non pour le travail ${ }^{30}$." La nuit peut alors avoir trois fonctions: sommeil, plaisir, travail. Napoléon (encore lui!) travaillait, paraît-il, la nuit. Goethe, déguisé, se promène dans les rues. "A la nuit répond le goût du masque et du travestissement. Toutes les rencontres sont possibles, lorsqu'on est autres ${ }^{31}$." La nuit, c'est aussi l'attente, analogue de cette autre nuit qu'est 
la mort. Et cette attente est une nuit de l'esprit, le moment offert pour se libérer par l'écriture. Si Éthier-Blais ne travaille pas la nuit, il lui reste le choix du sommeil ou des plaisirs. II peut aussi par la fenêtre regarder les astres qui composent le zodiaque, s'inquiéter de leur ordre, de leur influence. II peut attendre là le moment du chaos final mais s'il veut apaiser son esprit, il peut aussi prendre un livre ou écouter une pièce de musique.

Les textes qui composent le Dictionnaire sont en majorité des textes de six ou huit pages (dans dix-huit cas). Or le texte sur la "Lecture" en comporte seize (deux fois huit) et l'autre sur la "Musique", douze (deux fois six). Tout cela n'est que coïncidence, sauf que la lecture et la musique jouissent d'un traitement particulier. C'est dire leur importance dans la vie d'un homme décidé à chercher du côté de la fiction l'analgésique qui permet de supporter la vie. Dans ces pages, Éthier-Blais aime. Au-delà de la préciosité des propos, le livre devient corps et la musique âme: «l'écrivain fait corps avec son livre, le lecteur avec lui »; et plus loin: “j'écris. Pourtant, c'est la musique qui m'inspire ${ }^{32}$ ". Cette vie frêle, "en sursis", caricature de la vraie vie est le lot des poètes. Les deux activités viennent, paradoxalement, appuyer le fait de n'avoir rien à dire. Ce qui importe alors, c'est d'unir sa voix à celle des autres. II n'existe plus d'auteurs, ni de livres ni de musiques mais l'Auteur, le Livre et la Musique.

Quel prix faut-il payer pour aller dans ce lieu qui est tantôt Ciel, tantôt Enfer? Éthier-Blais ne le dit pas. Il laisse seulement entendre que c'est parfois (souvent) coûteux: "Quelle scie que cette vie, mon Dieu, quelle scie $^{33 !}$ »

\section{De l'effigie au masque mortuaire}

Et c'est ce qui me faisait souhaiter plutôt de mourir au loin, dans je ne sais quel accident, d'une mort rapide, loin des miens, comme souhaitait aussi Montaigne, sans témoins prêts à attacher à ces derniers instants une importance que je me refusais à leur reconnaître. Oui: sans autres témoins que de rencontre et anonymes.

\section{André Gide}

Lire suppose toujours qu'on se tienne à distance du livre, qu'on le manipule. Ouvrir, fermer, poser là. Au hasard des interruptions obligatoires mais aussi dans une sorte de rapport à l'ensemble, au rituel qui consiste à palper le corps du livre, à sonder la reliure, à examiner les moindres détails. Le toujours pompeux "Achevé d'imprimer sur papier..." Ici, c'est du «Val-de-Brôme des papeteries Eddy, Hull». Quelle antithèse! Le “dépôt légal » ne fait qu'appuyer “Le Conseil des arts..." subventionneur. La dédicace absente force la création de celle que l'on suppose: «à moimême» ou «à $X »$. Le plus étonnant demeure la photographie de Jean Éthier-Blais. 
Dans le Manteau de Rubén Dario ${ }^{34}$, l'auteur était correctement identifié de face, assez pour qu'on puisse le reconnaître à la table d'un petit restaurant italien. Ici, un profil de la figure qui regarde vers la droite. La tête se perd dans l'ombre. Le nez, le menton connotent quelqu'empereur romain en plein "floruit". Comme une pièce de monnaie. Et pourtant non. Les yeux sont (presque) fermés et curieusement la barbe pointe sur un visage qui devrait être rasé de près. Le masque mortuaire se superpose à l'effigie. L'obole présentée à Charon porte le portrait du mort. Vivant précautionneux! «Le quand? du passé se résorbe. Seul demeure celui du futur qui trébuche dans le vide, là où il n'y a plus ni questions ni réponses. Le silence seul face à un œil implacable et fermé ${ }^{35}$. »

La mort plane. "Quand?" La question est plus tragique que la réponse. "Quand mourrai-je? A cinquante-quatre ans, comme lui (mon père), afin d'accomplir dans sa totalité mon destin mimétique ${ }^{36}$. " La vie individuelle qui, par osmose, se transmet aux objets préférés, vient aussi les marquer du même tremblement. La “Table», lieu par excellence de l'écriture. et de la bonne chère, est vouée à devenir la dernière table de nuit, chargée de tous ces objets-signes de la mort que sont les médicaments, le thermomètre. Même le «Gant» n'y échappe pas. Masque de la main, peau des os, lui qui, un jour, habillait l'organe de la pensée, devient dépouille: «On les jette, comme plus tard on jettera notre dépouille ${ }^{37}$." Le «Wagon» devient aussi figure. II ne relie plus les villes et les gens. II ne provoque plus l'attente, la joie. II ne met plus l'accent sur les voyageurs: «Un jour, le train n'avance plus. Un voyageur descend. Bientôt ce sera moi. J'aurai vu la dernière gare ${ }^{38}$. " La mort est la véritable inconnue, la seule, le $« X »$. Elle vient gruger la vie. Un homme de cinquante ans est vieux s'il meurt à cinquante-quatre ans, jeune s'il devient centenaire. A moins de choisir, c'est-à-dire à moins de vouloir contrôler soi-même la réponse au «Quand? "

\section{Pour tout ce qui n'est pas dit}

L'ordre alphabétique efface tout, refoule toute origine.

Roland Barthes

Au terme de ce qui est une sorte de lecture à haute voix du Dictionnaire de moi-même, il reste les multiples insatisfactions qu'entraîne toute lecture. Les textes placés en tête de chacun des chapitres sont venus parfois souligner des tiraillements, des mises en parallèle d'auteurs ou même d'esprits. Évidemment une analyse plus rigoureuse aurait pu identifier chez Éthier-Blais un certain nombre d'idées-thèmes, de tournures stylistiques, etc. Ces analyses auraient eu le désavantage de limiter les intuitions, les découvertes en plus de faire subir un traittement rationnel à une œuvre qui ne l'est pas beaucoup. 
On peut quand même se demander ce qu'apporte à la littérature ce dernier livre d'Éthier-Blais. II était déjà annoncé dans Signets $/ 1{ }^{39}$. C'était même une sorte de promesse. Cela justifie pleinement son origine, du moins du point de vue de l'auteur. II n'en reste pas moins que ce livre a un caractère vieillot, précieux, qui sied mal au désespoir d'une partie de la littérature et aux recherches aventureuses de l'autre partie. L'univers littéraire d'Éthier-Blais est si universel qu'il ressemble à la mémoire d'un normalien. II en a aussi le manque de profondeur. L'anachronisme est une de ses vertus. Toutes ces femmes réelles ou fictives qui traînent encore les insignes de régimes passés, ces auteurs qui sont bons à citer, parce qu'ils n'ont plus d'importance, ces formules précieuses qui cultivent la surprise syntaxique, ces mots-bijoux sortis d'un écrin usé, tout cela apparaît comme une longue méditation sur des sujets éculés.

L'intérêt du Dictionnaire, sa vérité, semblent résider dans ce glissement continu vers la mort, une mort qui est refusée et que l'écriture cherche à neutraliser. La lecture se fait comme on demeure au chevet d'un mourant, dont le souffle de vie menace à chaque instant de s'interrompre. Ce témoignage d'une agonie ressentie dans tout l'être est probablement un exemple unique en littérature québécoise. Ses références sont raffinées, baroques comme Rubén Darío.

Malheureusement cette littérature est marquée du signe de la contradiction. D'une âme européenne, elle n'intéresse pas les Européens. Et ici, volontairement à l'écart du vécu québécois, sauf dans de rares cas et références, elle ne peut que glacer ceux qui voudraient savoir comment on peut être universel... En fait la prétention à l'universalité est un rêve provincialiste.

D'aucuns reprocheront à cet ouvrage son idéologie. Ils parlent d'extrême-droite, d'élitisme et de bourgeoisie. Il ne s'agit pas de ça. II y a bien pour Éthier-Blais, une gauche et une droite. Il n'est ni d'un côté ni de l'autre... parce qu'il s'y trouve déjà d'autres personnes et le seul centre acceptable, c'est celui où il est seul. Anachronie politique, anarchie d'un homme d'ordre, univers égoïste, racisme occidentalo-européen.

Que vous apprend cet essai sur l'auteur? Peu de choses. Rien de ce dont il a pu être témoin à Hanoï au moment de la conférence de Genève, rien de la IVe République française et de ses remous, rien de l'aprèsguerre. Les visites à l'Allemagne se sont faites dans un contexte apolitique. Si nous y trouvons une condamnation (un peu ambiguë) de Trudeau et autres fédéralistes, ce n'est que pour être plongés dans une diatribe contre la jeuñesse en général et la nôtre en particulier.

Enfin, le faux hasard de l'alphabet et de ses choix ne fait qu'imposer un cadre plus étroit que la simple évocation des lettres. Le choix des figures n'est ni unique ni univoque. Éthier-Blais a écrit une ville dans laquelle il se promène vingt-six fois travesti. Le plaisir du texte ne consiste pas à lever les masques mais à suivre le parcours. Celui qui s'intéresse à la 
littérature a toujours du temps à donner pour la recherche d'un temps perdu.

\section{Gilles Thérien}

Université du Québec à Montréal

1. Aux Éditions La Presse, Montréal, 1976. Nous utiliserons dorénavant l'abréviation suivante: $D d m m$.

2. Publié chez Grasset et au Cercle du livre de France en 1968.

3. Ddmm, p. 190.

4. Ibid., p. 12.

5. Ainsi se trompe-t-il d'âqe ou de date en calculant les nombres mystiques de la vie de son père. 1884-1934 couvre un espace de cinquante ans et non de cinquante-quatre! Ddmm, p. 138.

6. Ddmm, p. 190.

7. Ibid., p. 191.

8. Ibid., p. 70.

9. Ibid., p. 7.

10. Ibid., p. 10.

11. Ibid., p. 8.

12. Ibid., p. 196.

13. Ibid.

14. Ibid., p. 197.

15. Introduction à la psychanalyse, Paris, Payot, "Petite Bibliothèque Payot", p. 355.

16. Ibid., p. 72.

17. $D d m m$, p. 17.

18. Ibid., p. 40.

19. Ibid., p. 131 et p. 134.

20. Ibid., p. 136-137.

21. Ibid., p. 63.

22. Ibid., p. 67.

23. Ibid., p. 81.

24. Ibid., p. 82.

25. Ibid., p. 87.

26. Ibid., p. 149.

27. Ibid., p. 154.

28. Ibid., p. 161.

29. Ibid., p. 129.

30. Ibid., p. 117.

31. Ibid., p. 119.

32. Ibid., p. 91 et 105.

33. Ibid., p. 157.

34. Publié à Montréal chez HMH en 1974.

35. Ddmm, p. 144.

36. Ibid., p. 140.

37. Ibid., p. 58.

38. Ibid., p. 180.

39. Par un texte qui s'intitule «le Poids des choses " et se termine par ces mots: "Un jour, je raconterai cela, aussi." Ce texte contient presque tout l'alphabet élu dans le $D d m m$. 